\title{
LAS PSEUDOPARADOJAS GEOMÉTRICAS PARA POTENCIALIZAR EL CONCEPTO DE ÁREA EN EL SÉPTIMO GRADO
}

\author{
OF GEOMETRIC PSEUDOPARADOJAS TO POTENTIALIZE \\ THE CONCEPT OF AREA IN THE SEVENTH GRADE
}

\section{DE PSEUDOPARADOJAS GEOMÉTRICOS PARA POTENCIALIZAR \\ O CONCEITO DE ÁREA NO SÉTIMO GRAU}

\author{
CARLOS ALBERTO BERRÍO PÉREZ* \\ MARY FALK DE LOSADA** \\ OSVALDO JESÚS ROJAS VELÁZQUEZ***
}

\section{RESUMEN}

En la investigación se analiza la incidencia que tiene la aplicación del enfoque teórico de la resolución de problemas, para motivar el estudio de la conservación del área, a través del uso positivo de las pseudoparadojas geométricas, en los grados séptimos de la Institución Educativa Distrital Cedid Ciudad Bolívar. Este estudio se sustenta en la visualización matemática y en la Comunidad de Práctica de Wenger (2002), para la comprensión de dichas pseudoparadojas. En el trabajo se diseñan actividades, donde se integra los contenidos de números enteros, fracciones y geometría, para explicar coherentemente las inconsistencias de estas pseudoparadojas. Estas actividades se realizan con los estudiantes de dos grupos del grado séptimo, a través de problemas retadores, donde se les posibilita hacer uso de su creatividad para desarrollarlas y discutirlas en pequeños equipos de trabajo. Con su implementación se promueven espacios académicos nuevos para los estudiantes, pues le permite lograr un mejor desempeño en el rendimiento académico de las clases de matemáticas y desarrollar óptimas competencias junto con el progreso de las relaciones sociales al interior del aula.

Palabras claves: figuras geométricas, resolución de problemas, visualización, pseudoparadojas, conservación del área.

\section{ABSTRACT}

The research analyses the incidence of the application of the theoretical approach to problem solving, to motivate the study of the conservation of the area, through the positive use of geometric pseud paradoxes, in the seventh grades of the Educational Institution of the Distrital Cedid Ciudad Bolivar. This study is based on the mathematical visualization of Wenger and Community of Practice (2002) to understand these pseud paradoxes. Work activities are designed where whole contents, fractions and geometry are integrated to consistently explain the inconsistencies of these pseud paradoxes. These activities are conducted with two groups of students from seventh grade through challenging problems, where they are possible to use their creativity to develop and discuss them in small teams. With its implementation, new academic spaces are promoted for students, as it allows them to generate better academic performance of math classes and develop optimal skills along with the progress of social relationships within.

Keywords: geometric shapes, problem solving, visualization, pseudparadoxes, conservation of the area.

\footnotetext{
* Estudiante del programa de Maestría y Doctorado de Educación Matemática. Universidad Antonio Nariño (UAN), Bogotá, Colombia. E-mail: paisacedid@gmail.com. Orcid: https://orcid.org/0000-0001-5312-1664

** Docente del programa de Maestría y Doctorado de Educación Matemática. Universidad Antonio Nariño (UAN), Bogotá, Colombia. E-mail: mariadelosada@gmail.com. Orcid: https://orcid.org/0000-0002-6380-0481

*** Docente del programa de Maestría y Doctorado de Educación Matemática. Universidad Antonio Nariño (UAN), Bogotá, Colombia. E-mail: orojasv69@uan.edu.co. Orcid: https://orcid.org/0000-0002-5387-0435
} 


\section{RESUMO}

A presente investigação visa analisar a incidência da aplicação da abordagem teórica de resolução de problemas, através do uso positivo de pseudoparadoxos geométricos, nas sétimas séries da Instituição Educacional do Distrito de Cedid Ciudad Bolivar, a fim de motivar o estudo da conservação da área. Este estudo baseia-se essencialmente na visualização matemática e na Comunidade de Prática de Wenger (2002), para a compreensão desses pseudoparadoxos. No trabalho são propostas atividades, cujo conteúdo de números inteiros, frações e geometria são integrados, objetivando explicar de forma coerente as inconsistências desses pseudoparadoxos. As referidas atividades são realizadas com o corpo discente de duas turmas da sétima série, através de situações-problema, onde os alunos poderão usar a criatividade para elucidá-los e discuti-los em pequenas equipes. Com a implementação destas atividades, novos espaços acadêmicos serão construídos para os alunos, posto que otimizará o desempenho do discente, possibilitando, assim, melhor rendimento nas aulas de matemática, além do fato de que promoverá o desenvolvimento de habilidades sociocultural e as relações humanas em sala de aula.

Palavras-chave: figuras geométricas, resolução de problemas, visualização, pseudoparadoxos, conservação da área.

\section{INTRODUCCIÓN}

Aprender a conocer, a hacer, a convivir y a ser, constituyen pilares básicos del aprendizaje que la educación debe crear y desarrollar (DELORS, 1997). En las escuelas actualmente se implementan metodologías, se introducen nuevas tecnologías, se diseñan diferentes formas de organizar la enseñanza y evaluar el aprendizaje de la Matemática, con el que se contribuye a estos pilares. A pesar de todo, persisten dificultades de contenidos, en temáticas tan básicas como geometría, trabajo con fracciones y números enteros, que limitan el logro de ellos.

El proceso de enseñanza y aprendizaje de la matemática, específicamente la conservación del área, a través de pseudoparadojas geométricas en la Educación ha ocupado a investigadores, tanto nacional como internacionalmente. Los resultados alcanzados se valoran en diferentes reuniones y congresos: Congreso Internacional de Educación Matemática (ICME), en el Congress of the European Society for Research in Mathematics Education (CERME), Conference of the International Group for the Psychology of Mathematics Education (PME), y el Encuentro de Geometría y sus Aplicaciones en Colombia, entre otros. En estas reuniones se ofrecen cursos, conferencias y se presentan ponencias que reflejan las dificultades y avances de las temáticas referidas en la escuela.

En el ICME 13 (2016) en el Grupo de Estudio de Temas, TSG 13 se aborda "La enseñanza y el aprendizaje de la geometría en nivel secundario", haciéndose referencia a las conexiones entre la geometría, las prácticas matemáticas y los procesos, tales como argumentación y visualización.

En el CERME 10 (2017) en el grupo temas de trabajo (TGW 4) se aborda la enseñanza aprendizaje de la geometría, específicamente la construcción de conceptos, la manipulación de instrumentos y la visualización. En la conferencia del PME (2018) desarrollada en Rancagua, Chile, se llevaron a cabo contribuciones relacionadas con el conocimiento geométrico inicial, la visualización, y las acciones sobre objetos mentales y materiales.

Específicamente en el XIII Encuentro de Geometría y I de Aritmética en el 2002, se exponen las paradojas en la matemática. En este congreso se aportan elementos de las implicaciones que han tenido las paradojas en la evolución de las matemáticas, a través de exposiciones que más han influenciado en la historia de la matemática. Estos criterios dados en los anteriores congresos, constituyen algunos de los elementos que muestran la pertenencia y actualidad de la investigación. 
En la literatura científica consultada, Kondratieva (2007) expresa que las paradojas tienen por objeto dar a conocer una inconsistencia o contradicción y llevan un reto especial. El uso de los términos de paradojas y pseudoparadojas se precisan para los propósitos de la investigación en el marco teórico.

En resumen, esta investigadora aporta estrategias y alternativas, donde demuestra las implicaciones del uso de las pseudoparadojas en la enseñanza aprendizaje de las matemáticas, pero en el ámbito universitario, no se evidencia una reflexión para su aplicación en la Educación Básica. En la práctica se carece de una estrategia que permita integrar los siguientes contenidos en el aula: geometría, trabajo con fracciones y números enteros. Además, es de destacar que se carecen de investigaciones sobre esta temática en la educación básica colombiana.

Por otra parte, al desarrollar los contenidos programáticos en la Institución Educativa Distrital CEDID Ciudad Bolívar, se encuentra que están organizados de la siguiente manera: primer y segundo periodo, números enteros; tercer periodo fracciones, en paralelo con la geometría. Esto evidencia que no hay una integración de dichas temáticas.

Se precisa como situación, en el área de la geometría, la confusión de los estudiantes con relación al perímetro y el área. En este proceso es limitado el trabajo de transformaciones en figuras geométricas en otras, para dar cuenta de la conservación del área. También es deficiente la enseñanza de la geometría enfocada en los procesos de comparación, descomposición y recomposición de las figuras geométricas (ROJAS 2009, BERRÍ0, 2015).

Con relación al desarrollo de los números enteros y las fracciones, sólo se evidencian propiedades aritméticas, mas no una construcción asociada para la comprensión de tales propiedades. La suma, resta, multiplicación y división es lo primordial para dar cumplimiento al programa y a las exigencias del Ministerio de Educación Nacional de Colombia (MEN).

A través del análisis de las investigaciones realizadas en el estado del arte (que abordan dificultadas en el proceso aprendizaje del concepto de área), de la observación a clases en el grado séptimo de la Institución Educativa Distrital CEDID Ciudad Bolívar y de las experiencias de los investigadores, se constata las siguientes insuficiencias:

- Limitados conocimientos geométricos y aritméticos.

- Se carece de una comprensión adecuada del concepto de área.

- Deficiente la enseñanza de la geometría enfocada en los procesos de comparación, descomposición y recomposición de las figuras geométricas.

- Escasa motivación en los estudiantes por la clase de matemáticas.

Las valoraciones anteriores y el estudio epistemológico inicial realizado precisan determinar como objetivo de la investigación elaborar una propuesta de actividades para la enseñanza y el aprendizaje de la conservación del área, donde se integre la geometría, las fracciones y los números enteros, usando pseudoparadojas geométricas con estudiantes de grado séptimo, de la Institución Educativa Distrital CEDID Ciudad Bolívar.

\section{MARCO TEÓRICO}

En la investigación se asumió como sustento de las actividades los referentes sobre paradojas, la teoría de la resolución de problemas, la visualización matemática y la Comunidad Práctica de Wenger (2001). 
Con respecto a las paradojas en la literatura científica consultada se destacan: Movshovitz-Hadar y Hadass (1990,1991), Kleiner y Movshovitz-Hadar (1994), Sullivan y Panasuk (1997), Castro y Pérez (2002), Kondratieva (2007), Macho (2012), Farlow (2014), entre otros.

Dado que las paradojas en matemáticas generan crisis y reestructuraciones en sus teorías (KLEINER y MOVSHOVITZ-HADAR, 1994), en ningún momento se pretendió el análisis de paradojas en este sentido con los estudiantes. La intención de la investigación consistió en traer situaciones al aula de tal modo que se mejorara la comprensión de conceptos matemáticos. Por este motivo, se presenta la definición de paradoja dada por Kondratieva (2007), que para los propósitos de la actual investigación se asumió como pseudoparadoja. Lo que interesa es el aspecto didáctico para la aplicación de las actividades a desarrollar con los estudiantes.

Kondratieva (2007, p. 1) propone una definición de particular interés para los propósitos de esta investigación, pues declara que "... una paradoja en el sentido amplio, es algo inesperado, surgido repentinamente, una declaración que se ve mal y contradictoria...su presencia facilita el proceso de comprensión de las cosas en un intento para corregir un error y hacerlo con sentido".

Se comparte las ideas de los investigadores Kondratieva (2007), Kleiner y Movshovitz-Hadar $(1990,199)$, y Castro y Pérez (2002), al plantear que las pseudoparadojas sorprenden, provocan, exasperan, llaman la atención y trastornan el pensamiento matemático del que está expuesto a ellas. En el proceso de la elaboración y desarrollo de las actividades de la presente investigación se asumieron las recomendaciones sugeridas por Kondratieva (2007, p. 7), las cuales son:

- "Enseñar paradojas para ilustrar un punto específico.

- Permitir a los estudiantes algunas veces tiempo para pensar por su cuenta.

- Involucrar a los estudiantes en una discusión. Hacer que ellos desarrollen sus conclusiones, llevándolos a una resolución.

- El objetivo es desequilibrar al alumno en una manera estéticamente valiosa, y a continuación, hacer uso de su curiosidad natural".

Por tales motivos, el uso de pseudoparadojas en las clases de matemáticas se debe retomar, ya que:

- Cautivan, divierten, exasperan, seducen, despiertan la curiosidad y motivan, cuestiones esenciales para lograr un proceso de enseñanza aprendizaje robusto en contenidos matemáticos (KLEINER y MOVSHOVITZ-HADAR, 1994).

- Generan conflictos cognitivos y motivan para lograr un aprendizaje constructivo.

- Facilitan el proceso de comprensión de los contenidos matemáticos.

- Pueden mejorar el conocimiento de la heurística en la resolución de problemas.

- Pueden provocar un cambio de actitud con respecto a la enseñanza aprendizaje de las matemáticas.

En cuanto a la teoría de resolución de problemas se destacan Polya (1973), Kilpatrick (1969), Schoenfeld (1992, 2014), Sriraman \& English (2010), Pochulu y Rodríguez (2012), Liljedahl y Santos-Trigo (2019), entre otros, los cuales aportan definición de problemas, de resolución de problemas y estrategias para su resolución. En la investigación se asumió la definición de problema dada por Polya (1981, p.117), donde expresa que "... significa buscar de forma consciente una acción apropiada para lograr un objetivo claramente concebido, pero no alcanzable de forma inmediata". También se asumió las cuatro fases para la resolución de problemas: "Comprender el problema, diseñar un plan, llevar a cabo el plan y por último, mirar hacia atrás” (POLYA, 1973, p. 5-16). 
Las actividades estuvieron conformadas por problemas retadores, Pérez (2004, p. 12) expresa que estos "... exigen la integración de conceptos relacionados y el establecimiento de nexos con otras áreas de la matemática, para lograr un dominio y una comprensión profunda de la matemática elemental, sin tratar de extender los conocimientos de los estudiantes hacia conceptos propios de la matemática superior". Es de precisar que los problemas que generen motivación e interés en los estudiantes deben incluir las siguientes características, "... que sea una situación que estimula el pensamiento, que sea interesante para el alumno, y que la solución no sea inmediata" (Falk, 1980, p. 16).

Las relaciones descritas anteriormente, tales como la indagación, el razonamiento, la exposición de su actuación, la integración de conceptos, y otros como la motivación e interés, permiten lograr una comprensión profunda de la matemática en la Educación Básica, y surgen del progreso de las actividades que se proponen en el desarrollo de esta investigación.

La visualización en matemáticas es considerada por varios investigadores de la educación matemática como una herramienta poderosa que, si se emplea adecuadamente, posibilita un adecuado aprendizaje de ideas y conceptos matemáticos. Se destacaron, para los propósitos de la investigación, Zimmermann y Cunningham (1991), De Guzmán, (1996) y Arcavi (2003), quienes han aportado referentes teóricos y estrategias.

En la presente investigación se asumió la definición de Zimmermann y Cunningham (1991, p. 3), quienes plantean que “... la visualización matemática es el proceso de formar imágenes (mentalmente, con lápiz y papel o con ayuda de materiales 0 tecnologías) y de utilizar estas imágenes de manera efectiva para el descubrimiento y la comprensión matemática". En la investigación se asumió esta definición, pues se enfatizó en la visualización como un recurso que propicia el descubrimiento y la comprensión matemática, elementos importantes para la resolución de las pseudoparadojas.

Con relación a la teoría de comunidad práctica de Wenger, se admitió que "[...] son grupos de personas que comparten una preocupación, un conjunto de problemas o una pasión sobre un tema, y que profundizan en su conocimiento y experiencia en esta área mediante la interacción de forma permanente" (WENGER, MCDERMOTT y SNYDER, 2002, p. 4). Estas comunidades comparten una estructura básica, pues es una "... combinación única de tres elementos fundamentales: un dominio de conocimiento, una comunidad de personas y una práctica común" (Idem, p. 27). Es de destacar que una práctica exitosa depende de un equilibrio entre las actividades, la exploración de ideas y la producción de un conocimiento conjunto.

\section{METODOLOGÍA}

La investigación se realizó con un enfoque de investigación mixto y un diseño de investigación acción. Este diseño “... constituye un proceso de reflexión-acción-cambio-reflexión, por y para el mejoramiento de la práctica del docente, mediante la participación activa de este, dirigido a superar los problemas y las necesidades del aula, la escuela y la comunidad, posibilitando el diálogo entre teoría-práctica-teoría" (MINERVA, 2006, p. 116).

La población estuvo constituida por estudiantes de grado séptimo y la muestra 73 estudiantes de los grupos 703 y 704 de la Institución Educativa Distrital CEDID Ciudad Bolívar. Los estudiantes que participaron de las actividades tenían entre 10 y 12 años. La institución educativa está ubicada en un estrato socioeconómico de nivel 1. La construcción del conocimiento geométrico en el grado séptimo y la edad, no fueron un impedimento para que los estudiantes avanzaran de la mejor manera en explicar coherentemente las pseudoparadojas. Fue todo un reto para ellos explicar estos dos hechos 
geométricos tan extraños, ya que generalmente realizaron sin dificultad transformaciones en las figuras para llevar a cabo cálculos de áreas y donde tenían presente el principio de la conservación de ésta.

En la investigación se combinaron métodos y técnicas de investigación científica, en un nivel teórico (Histórico-lógico y análisis-síntesis) y empírico (Observación participante y encuesta). Estos últimos se aplicaron, con el objetivo de obtener información sobre las dificultades y el éxito de aplicar las actividades, acerca del proceso de enseñanza aprendizaje de la geometría, a través de las pseudoparadojas en el grado séptimo. También se utilizan los métodos Matemáticos estadísticos (procedimiento del cálculo porcentual) con el fin de procesar la información obtenida en los diferentes momentos de la investigación.

Se presentaron dos actividades para la enseñanza y aprendizaje de la conservación del área, donde se integraron la geometría, las fracciones y los números enteros, usando pseudoparadojas geométricas. Las actividades propuestas se estructuraron en título, objetivo, materiales didácticos y desarrollo de la actividad. A continuación, se presentan las actividades "Un triángulo mágico" y "Un cuadrado extraño".

Título: Un triángulo mágico.

Objetivo: Explicar las inconsistencias que aparecen en la pseudoparadoja geométrica que evidencia la no conservación del principio del área.

Materiales didácticos Papel cuadriculado de cuadritos 1x1, lápices de colores y reglas.

Desarrollo de la actividad:

En la figura de la izquierda se muestra un triángulo asociado a determinados puntos reticulares y en la figura de la derecha se muestra otro triángulo con una reorganización diferente, pero con las mismas piezas. Analice y responda las siguientes interrogantes.
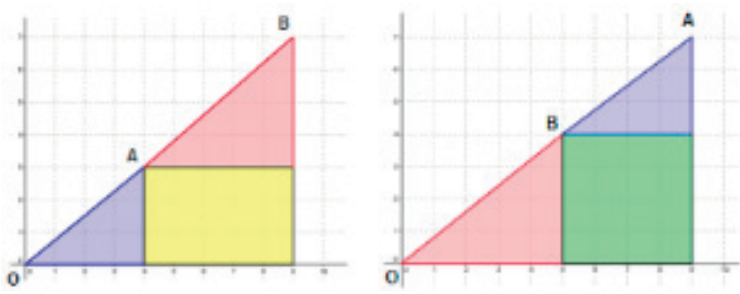

a) ¿Cuáles son los puntos reticulares que definen los vértices de estos triángulos?

b) ¿Cuáles son los puntos reticulares del punto $\mathrm{A}$, en primer triángulo?

c) ¿Existe un punto reticular sobre la prolongación del segmento $\mathrm{OA}$ en el primer triángulo con coordenadas $(8,6)$ ?

d) ¿Existe un punto reticular sobre la prolongación del segmento $\mathrm{OA}$ en el primer triángulo con coordenadas $(12,9)$ ?

e) ¿Pertenece el punto reticular $(9,7)$ a la prolongación del segmento $0 A$ en el primer triángulo?

f) ¿El segmento con extremos reticulares $(4,3)$ y $(9,7)$ pertenece a la prolongación del segmento

$\mathrm{OA}$ en el primer triángulo?

g) ¿Puede concluirse que $O A B$ es un segmento en el primer triángulo?

h) ¿En la primera figura los segmentos $O A$ y $A B$ están definidos por dos razones diferentes?

i) ¿Cuáles son las razones que definen a estos segmentos?

j) ¿Son las razones calculadas anteriormente equivalentes? 


\section{Problema retador.}

¿Por qué no se cumple "el principio de la conservación del área" en esta situación cuando se practican en estos triángulos las siguientes subdivisiones?
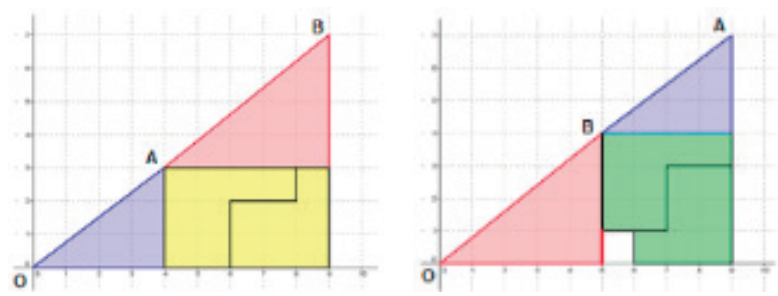

Título: Un cuadrado extraño.

Objetivo: Explicar las inconsistencias que aparecen en la pseudoparadoja geométrica que demuestra la no conservación del principio del área.

Materiales didácticos: Papel cuadriculado de cuadritos 1x1, lápices de colores y reglas.

Desarrollo de la actividad:

Problema retador: un cuadrado extraño.

Se construye un cuadrado como el que aparece en figura de la izquierda, asociado a determinados puntos reticulares.
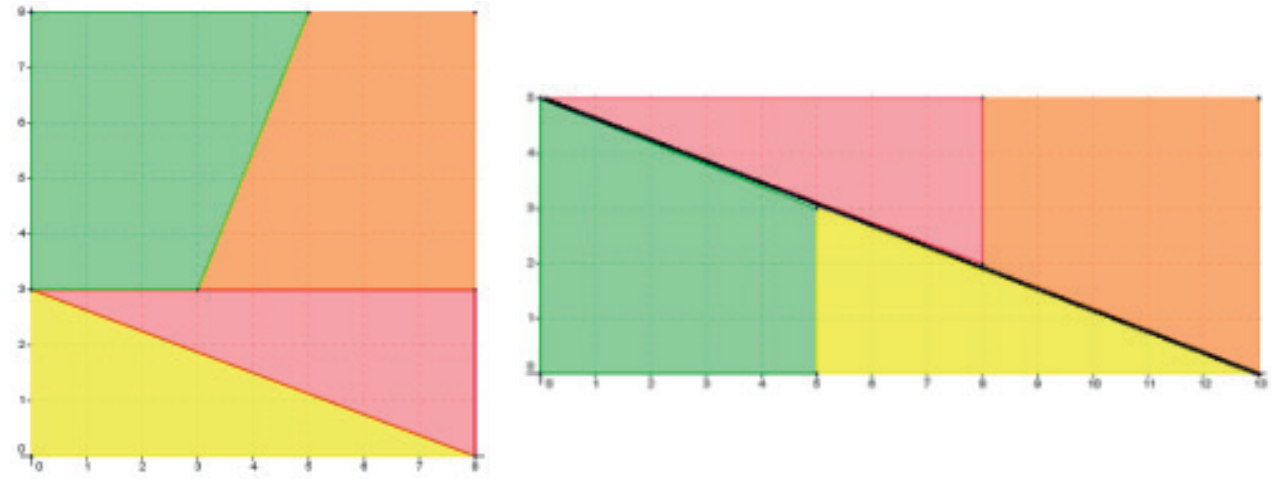

Este cuadrado se transforma en el rectángulo de la figura que se adjunta a la derecha. Explique:

a) ¿Por qué hay una ganancia de una unidad de área con sólo intercambiar las piezas que conforman el cuadrado?

b) ¿Por qué no se cumple "el principio de la conservación del área" en esta transformación?

Para la resolución de las actividades en el aula se conformaron grupos de trabajo de tres estudiantes cada uno. Se entregó la guía de cada actividad y comenzaron su desarrollo a nivel grupal. Los estudiantes mostraron interés y agrado al trabajar en cada una de las actividades.

La recolección de los datos se logró, con la resolución de la guía de trabajo, muestras fotográficas contentivas del trabajo grupal y de las mejores soluciones de los estudiantes. También, a través de videos que contienen el trabajo realizado por los estudiantes durante la resolución en el grupo y la socialización en el aula.

Para el procesamiento de la información se realizó una valoración de las actividades desarrolladas por los estudiantes, donde se realiza un análisis mediante tres categorías: la motivación por el aprendizaje, los logros y las dificultades encontradas en cada una de ellas. 


\section{RESULTADOS}

En el análisis de las actividades "Un triángulo mágico" y "Un cuadrado extraño", no se evidenció dificultad en la comprensión de las preguntas de cada actividad y mediante la socialización en los grupos de estudiantes, las soluciones surgidas se valoraban y se constataban en cada uno de ellos. El papel del docente y sus acciones estuvieron dirigidas a guiar el trabajo de los estudiantes.

La relación entre visualización matemática y resolución de problemas, en el contexto de las dos actividades propuestas, están dadas en los procesos que llevaron a cabo los estudiantes al resolver estas pseudoparadojas geométricas y en los resultados que se obtuvieron cuando los diferentes tópicos (Geometría, fracciones y números enteros) de la matemática del grado séptimo se relacionaron a partir de los problemas planteados, los cuales, apoyados en la visualización matemática, facilitaron la comprensión de las pseudoparadojas.

La visualización se puede emplear adecuadamente para permitir tanto construcción como transformación de imágenes mentales, posibilitando la comprensión, ordenación e implicación de nuevas relaciones, criterios estos necesarios en el proceso de resolución de problemas, que potencian los aprendizajes en los estudiantes.

La visualización no es sólo una herramienta para la comprensión de los conceptos matemáticos, sino que mediante su empleo se posibilita la comunicación de ideas en matemáticas (ARCAVI, 2003). Por otra parte, esta tiene que ver con las ideas y conceptos que son "[... ] representables intuitivamente, geométricamente, cuya utilización resulta muy provechosa" (DE GUZMÁN, 1996, p. 2). Tanto los criterios de Arcavi (2003) como los de De Gusmán (1996) son esenciales para el proceso de resolución de problemas geométricos basados en pseudoparadojas que se presentaron en la investigación.

El proceso de resolución de los problemas planteados en las actividades propició la experimentación, la exploración y la conjeturación, elementos estos que se enriquecen con el uso de la visualización matemática, la cual permitió la integración de la geometría, las fracciones y los números enteros.

\section{Resolución de la actividad 1: Un triángulo Mágico}

Se precisó como objetivo que los estudiantes pudieran explicar coherentemente las inconsistencias que aparecen en la pseudoparadoja geométrica, en donde aparentemente se evidencia la no conservación del principio del área empleando triángulos rectángulos. Aunque la pseudoparadoja es difícil de resolver, con la fundamentación matemática que se realizó con los estudiantes lograron explicarla satisfactoriamente.

Se presenta a los estudiantes como un triángulo mágico y se indaga el siguiente aspecto: ¿por qué no se cumple "el principio de la conservación del área" en esta situación?

Se puede evidenciar que los estudiantes formalizaron varias soluciones de este problema retador. También se valoró el esfuerzo de los grupos de estudiantes por explicar de la mejor manera la inconsistencia. Esto se observa en las figuras 1 y 2. 
Figura 1 - Solución grupo de estudiantes A.

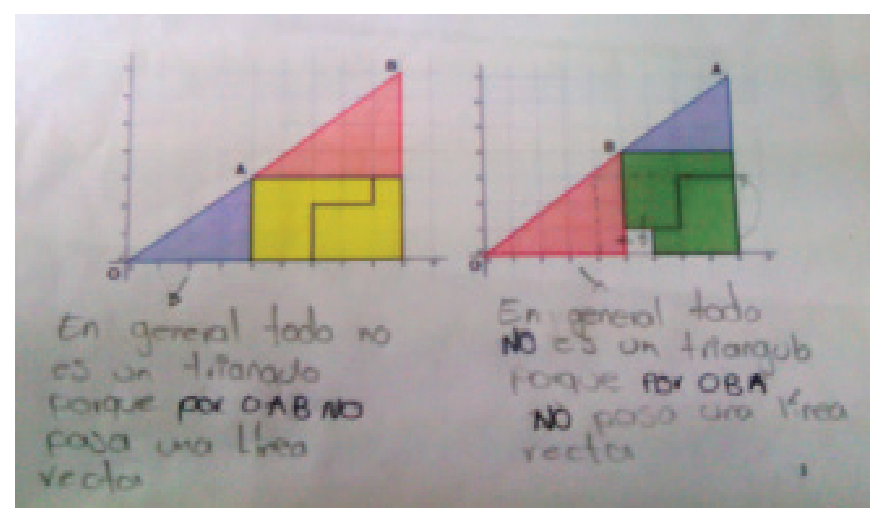

Fuente: Construcción de los autores.

Figura 2 - Solución grupo de estudiantes B.

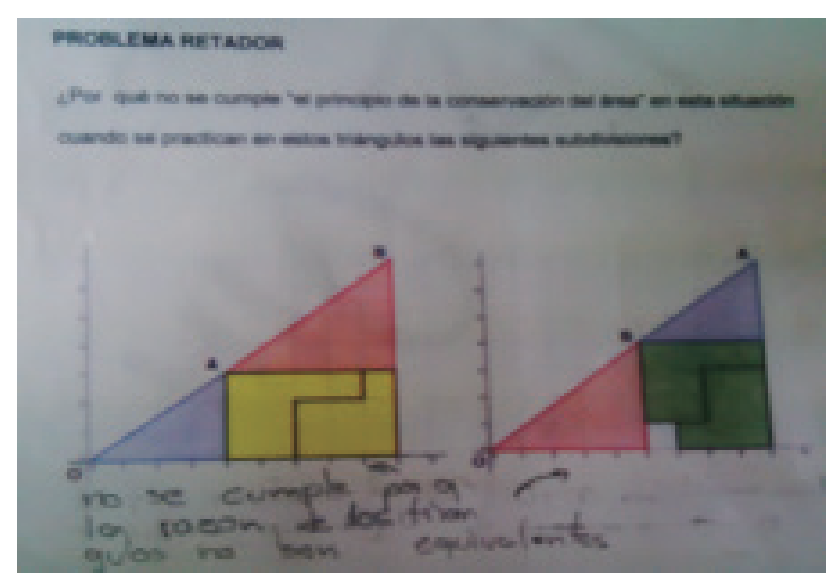

Fuente: Construcción de los autores.

Motivación por el aprendizaje. Esta actividad fue de gran expectativa para los estudiantes. Simplemente el hecho de mover unas piezas y recomponerlas para formar otro "triángulo igual" al original trae como consecuencia ganar una unidad de área. Esta es una "transformación" geométrica que debe ser estudiada con sumo cuidado. En la geometría conocida por el estudiante, el área de las figuras siempre se conserva.

Logros. La pseudoparadoja, motivó a que los estudiantes buscaran el error conscientemente y generaron conocimiento matemático construido por ellos mismos. Las ideas que surgieron aportaron y permitieron enfrentar la situación, en donde, la socialización fue fundamental.

Se logró un desarrollo en las formas de sustentación, pues se observa en las figuras 1 y 2 un esfuerzo por expresar las soluciones al problema planteado. Se avanzó a tal grado, que la conservación del área que aquí se puso en duda, ya pareció haber quedado establecida en los estudiantes, debido a que es independientemente de las transformaciones que se realicen en la figura. Es evidente 
la construcción del concepto de línea recta y de segmento de recta. También se observó como la razón entre segmentos y sus equivalencias han sido construidas para explicar la pseudoparadoja coherentemente. La actividad fue motivadora y se alcanzó el objetivo.

Dificultades. Hubo insuficiencias en los estudiantes, dado que la confianza en sus soluciones fue paulatinamente generándose con el desarrollo de la actividad. También se constataron limitaciones para escribir una solución coherentemente.

A pesar de las dificultades se percibió realmente lo mucho que se alcanzó con la aplicación de esta actividad en la construcción de conceptos e ideas en matemáticas por parte de los estudiantes.

\section{Desarrollo de la actividad 2: Un cuadrado extraño}

Se precisó como objetivo que los estudiantes pudieran explicar las inconsistencias en esta pseudoparadoja geométrica. Se indagó ahora ipor qué hay una ganancia de una unidad de área con sólo intercambiar las piezas que conforman el cuadrado? En el desarrollo de la actividad realizada por los estudiantes se evidenció el esfuerzo logrado por ellos para explicar las inconsistencias. En esta situación, se comprobó que hay varias formas en que los grupos de estudiantes proponen como explicar y entender las inconsistencias aquí presentadas. Esto es evidenciado en las figuras 3 y 4.

Figura 3 - Solución por un grupo de estudiantes.

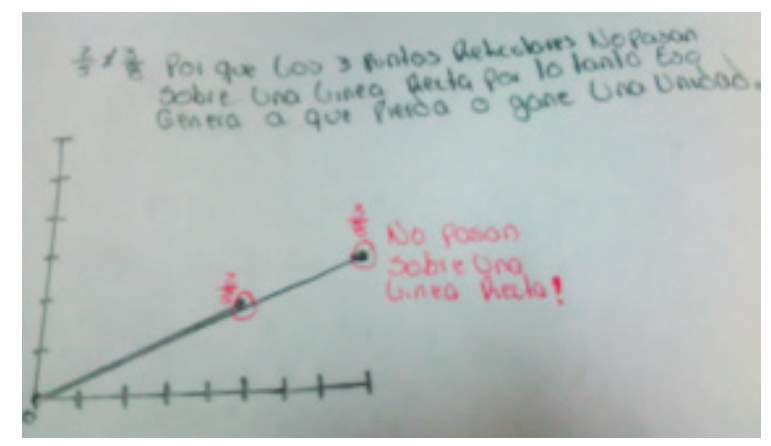

Fuente: Construcción de los autores.

Figura 4 - Solución por otro grupo estudiantes.

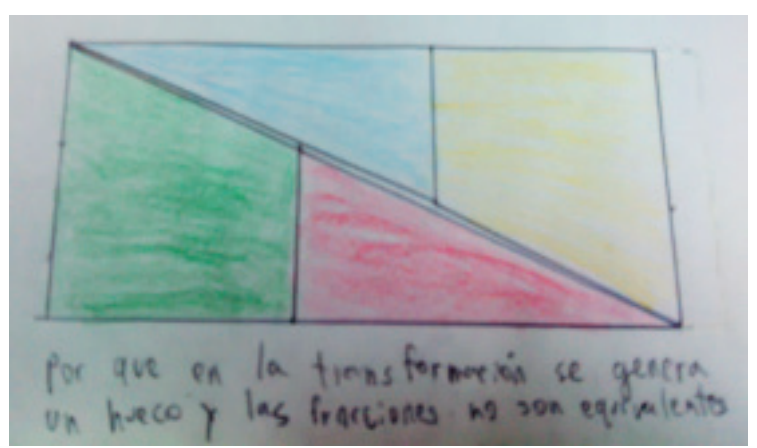

Fuente: Construcción de los autores. 
Motivación por el aprendizaje. En la literatura científica es conocida como El Cuadrado de Ball. Aquí se presentó a los estudiantes la pseudoparadoja como tal. Es importante estudiarla por el hecho de que al mover unas piezas y componerlas para conformar un rectángulo se gana una unidad de área.

Logros. En el intento por explicar su inconsistencia se refinaron conceptos matemáticos. Las ideas que allí brotaron aportaron y proporcionaron enfrentar esta situación, en donde la socialización fue fundamental en la actividad. Se avanzó en las formas de sustentación y explicación, pues de nuevo se observó un esfuerzo en las soluciones al problema planteado.

Se progresó a tal grado que la conservación del área es independientemente de las transformaciones que se realicen en toda figura. También se logró una construcción de las fracciones equivalentes para dar explicación a la pseudoparadoja y la comparación de la inclinación, para segmentos de recta 0 pendiente, fue un elemento decisivo.

Dificultades. De nuevo la confianza en sus soluciones fue gradualmente generándose con el desarrollo de la actividad. La manera como escriben no permitió realmente percibir lo mucho que se alcanzó con la aplicación de esta actividad.

\section{Valoración de los resultados obtenidos en la práctica escolar de la investigación a través de la encuesta de satisfacción}

Es de mencionar, como aparece en la introducción, que la mayoría de los estudiantes mostraban apatía por las clases de matemáticas, además se quejaban del papel del docente, en el sentido de solo enseñar fórmulas para luego realizar una serie de ejercicios de manera mecánica que están en los libros de texto. También expresaban abiertamente que nada les motiva bajo esta perspectiva. Por tales situaciones de aula se optó para contribuir a resolver las problemáticas antes mencionadas el empleo del análisis de pseudoparadojas. Las pseudoparadojas desempeñan en el aula de clases el papel de retar, incitar, invitar y motivar al estudio de algún contenido específico de las matemáticas elementales.

Para verificar los avances, se realizó una encuesta de satisfacción (ver Anexo 1) a los estudiantes, con el propósito de constatar el éxito de las actividades e indagar en aspectos fundamentales tales como la motivación y los logros alcanzados por ellos. Estos logros están dados en: el desempeño en el área de las matemáticas, las actividades propuestas constituyeron un reto, el desarrollo de un ambiente matemático y la motivación a realizar el trabajo de forma natural y autónoma. En la encuesta los estudiantes respondieron cinco preguntas en escala Likert, siendo cinco la máxima calificación y uno la mínima, el análisis de las encuestas se realiza en correspondencia a estas cinco escalas.

La aplicación de la encuesta de satisfacción a los estudiantes participantes consolida los resultados alcanzados en el desarrollo de las actividades, las cuales tienen que ver con la motivación que crearon las actividades por el estudio de la matemática. Con la aplicación de la encuesta se pudo constatar los avances en las dos problemáticas fundamentales planteadas por los estudiantes.

Al triangular el análisis realizado a cada una de las actividades con los resultados de la encuesta de satisfacción, se concluyó que son beneficiosos los logros obtenidos por los estudiantes al poder explicar la ganancia de una unidad de área en las actividades que se analizaron en el trabajo de investigación. El esfuerzo cognitivo por parte de los estudiantes al explicarlas, evidenció el empeño y la entrega, al sentirse retados por estas pseudoparadojas, tal como ellos mismos lo expresaban.

Se logró confirmar que este tipo de actividades desarrolladas en la investigación son aceptadas por los estudiantes del CEDID Ciudad Bolívar, así mismo, esto permitió que los estudiantes se apropiaron de una metodología para la resolución de problemas. 
En lo que sigue, se describen las respuestas que se obtuvieron después de finalizar las actividades en los grupos de la muestra. A continuación, en la Tabla 1, se muestra los resultados de la encuesta de satisfacción del grupo 703.

Tabla 1 - Resultados del grupo 703.

\begin{tabular}{|c|c|c|c|c|c|c|}
\hline CURSO 703 & & & & & & \\
\hline & 1 & 2 & 3 & 4 & 5 & CALIFICACION \\
\hline \multicolumn{7}{|l|}{ PREGUNTA } \\
\hline$a$ & 1 & 2 & 11 & 12 & 7 & \\
\hline b & 3 & 1 & 11 & 10 & 8 & \\
\hline c & 0 & 2 & 8 & 12 & 11 & \\
\hline d & 2 & 4 & 4 & 9 & 16 & \\
\hline e & 1 & 3 & 5 & 7 & 17 & \\
\hline
\end{tabular}

Fuente: Construcción de los autores.

¿Considera usted que las actividades desarrolladas motivan el estudio de la matemática?, el $3 \%$ de los estudiantes la calificaron con 1, el $6 \%$ de los estudiantes la estimaron con 2, el 33\% de los estudiantes la apreciaron con 3 , el $36 \%$ de los estudiantes la calificaron con 4 y el $21 \%$ de los estudiantes la consideraron con 5 (ver Figura 5). Los resultados muestran un 91\% de aceptación.

Figura 5 - Resultados del grupo 703.

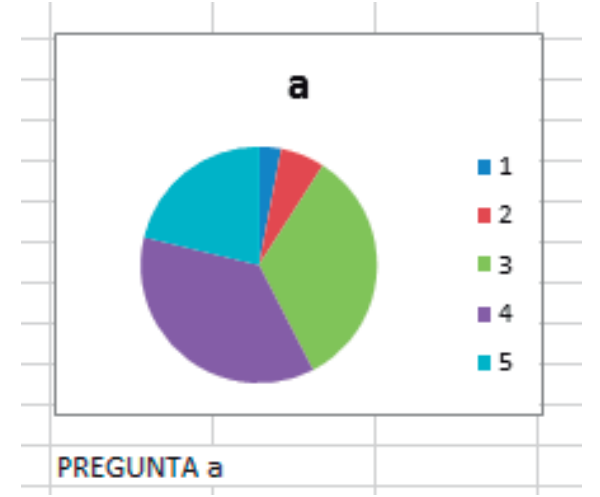

Fuente. Construcción de los autores.

¿Cree usted que su desempeño en el área de las matemáticas mejoraría si estas actividades se repitieran con frecuencia? En este sentido el $9 \%$ de los estudiantes la calificaron con 1, el $3 \%$ de los estudiantes la estimaron con 2, el $33 \%$ de los estudiantes la consideraron con 3, el $30 \%$ de los estudiantes la apreciaron con 4 y el $24 \%$ de los estudiantes la calificaron con 5 (ver Figura 6). El $87.8 \%$ de los estudiantes muestran aceptación de que una aplicación futura de actividades mejoraría su desempeño. 
Figura 6 - Resultados del grupo 703.

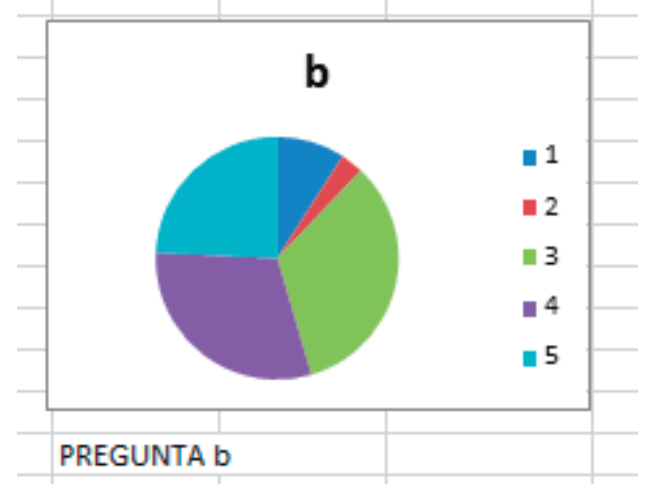

Fuente. Construcción de los autores.

¿Las actividades propuestas constituyeron un reto para usted? El $0 \%$ de los estudiantes la calificaron con 1, el $6 \%$ de los estudiantes la evaluaron con 2, el $24 \%$ de los estudiantes la ponderaron con 3 , el $36 \%$ de los estudiantes la apreciaron con 4, el $33 \%$ de los estudiantes la califican con 5 (ver Figura 7). Los resultados muestran un 94\% de aceptación.

Figura 7 - Resultados del grupo 703.

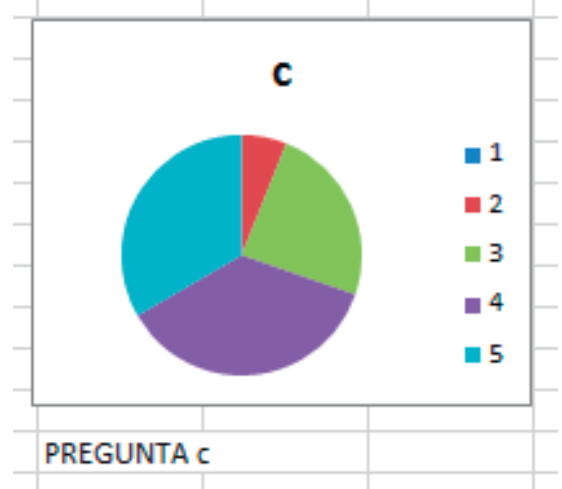

Fuente. Construcción de los autores.

¿Considera usted que se vivió durante el desarrollo de las actividades un ambiente matemático? En las respuestas el $6 \%$ de los estudiantes la calificaron con 1 , el $11 \%$ de los estudiantes la apreciaron con 2, el $11 \%$ de los estudiantes la consideraron con 3, el $26 \%$ de los estudiantes la evaluaron con 4 y el $46 \%$ de los estudiantes la estimaron con 5 (ver Figura 8). Los resultados muestran un 87.9\% de aceptación. 
Figura 8 - Resultados del grupo 703.

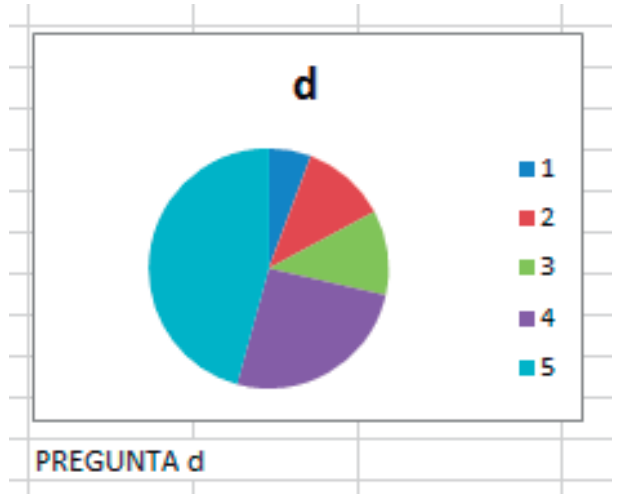

Fuente. Construcción de los autores.

¿Se sintió usted motivado a desarrollar los retos de forma natural y autónoma? Aquí el 3 \% de los estudiantes la calificaran con 1, el $9 \%$ de los estudiantes la apreciaron con 2, el $15 \%$ de los estudiantes la estimaron con 3, el $21 \%$ de los estudiantes la consideraron con 4 y el $52 \%$ de los estudiantes la calificaron con 5 (ver Figura 9). Los resultados muestran un 87.9\% de aceptación.

Figura 9 - Resultados del grupo 703.

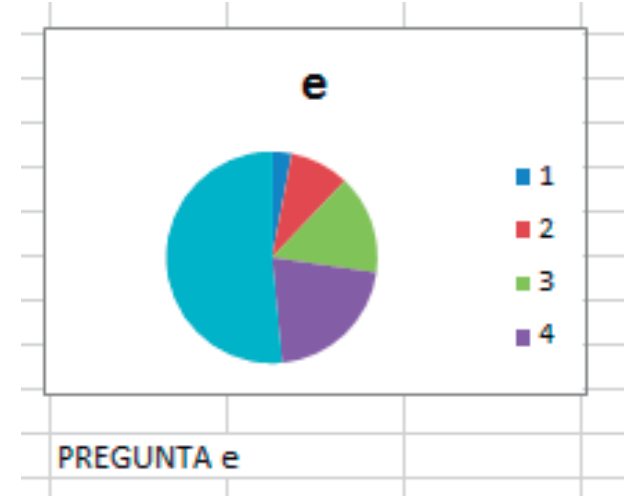

Fuente. Construcción de los autores.

A continuación, en la Tabla 2, se muestra los resultados de la encuesta de satisfacción del grupo 704. 
Tabla 2 - Resultados del grupo 704.

\begin{tabular}{|c|c|c|c|c|c|c|}
\hline CURSO 704 & & & & & & \\
\hline & 1 & 2 & 3 & 4 & 5 & CALIFICACION \\
\hline \multicolumn{7}{|l|}{ PREGUNTA } \\
\hline a & 0 & 0 & 4 & 18 & 16 & \\
\hline b & 0 & 1 & 6 & 11 & 20 & \\
\hline c & 2 & 2 & 5 & 11 & 18 & \\
\hline d & 0 & 1 & 6 & 13 & 18 & \\
\hline e & 1 & 2 & 10 & 17 & 8 & \\
\hline
\end{tabular}

Fuente: Construcción de los autores.

¿Considera usted que las actividades desarrolladas motivan el estudio de la matemática? En esta pregunta el $0 \%$ de los estudiantes la calificaron con 1, el $0 \%$ de los estudiantes la apreciaron con 2, el $11 \%$ de los estudiantes la consideraron con 3 , el $47 \%$ de los estudiantes la estimaron con 4 y el $42 \%$ de los estudiantes la valoraron con 5 (ver Figura 10). Los resultados muestran un 100\% de aceptación.

Figura 10 - Resultados del grupo 704.

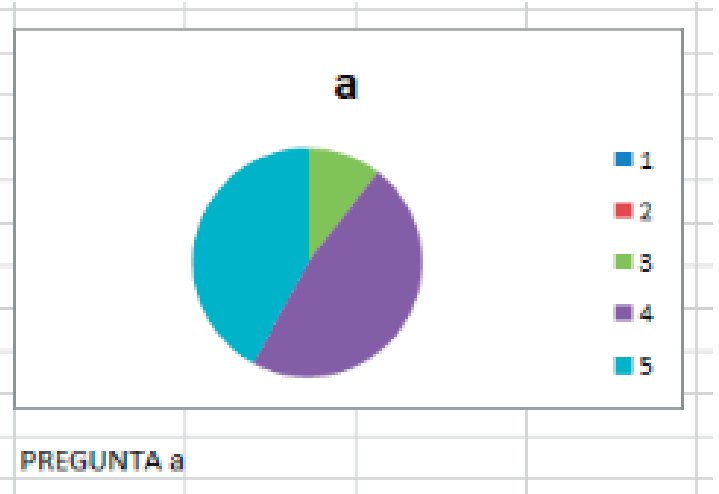

Fuente. Construcción de los autores.

¿Cree usted que su desempeño en el área de las matemáticas mejoraría si estas actividades se repitieran con frecuencia? Aquí el $0 \%$ de los estudiantes la evaluaron con 1, el $2 \%$ de los estudiantes la consideraron con 2, el $16 \%$ de los estudiantes la estimaron con 3, el 29\% de los estudiantes la califican con 4 y el $53 \%$ de los estudiantes la ponderaron con 5 (ver Figura 11). El $97 \%$ de los resultados muestran aceptación. 
Figura 11 - Resultados del grupo 704.

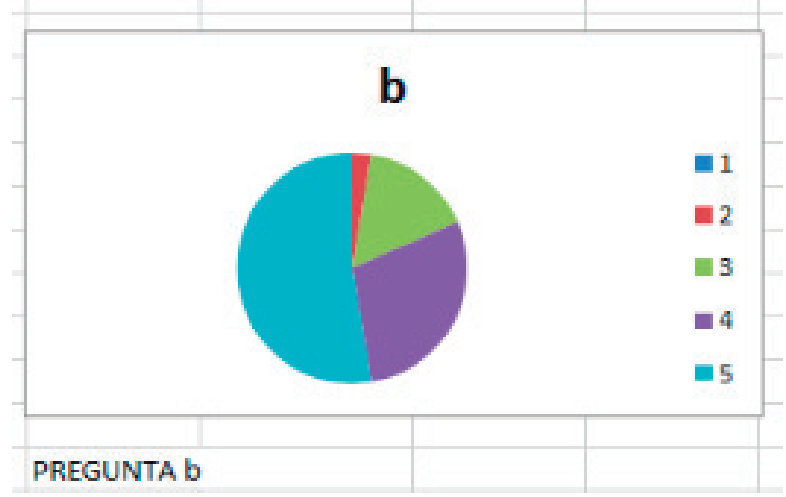

Fuente. Construcción de los autores.

¿Las actividades propuestas constituyeron un reto para usted?, el $5 \%$ de los estudiantes la califican con 1, el $5 \%$ de los estudiantes la calificaron con 2, el $13 \%$ de los estudiantes la evaluaron con 3, el $29 \%$ de los estudiantes la ponderaron con 4 y el $47 \%$ de los estudiantes la estimaron con 5 , como se muestra en la Figura 12. Los resultados muestran un 89\% de aceptación.

Figura 12 - Resultados del grupo 704.

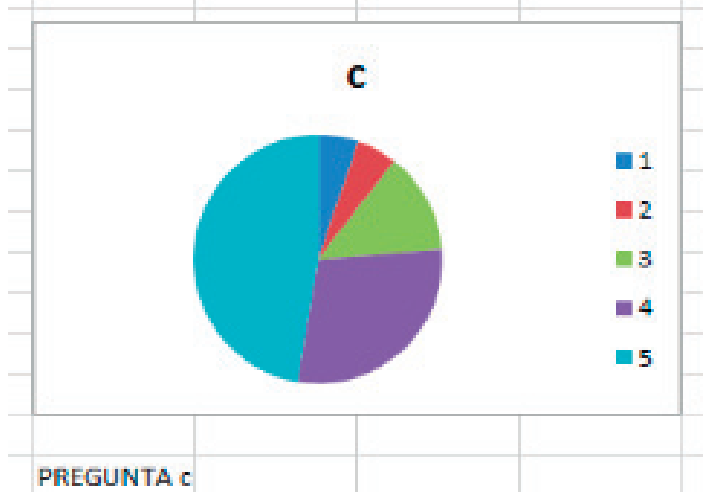

Fuente. Construcción de los autores.

¿Considera usted que se vivió durante el desarrollo de las actividades un ambiente matemático?, el $0 \%$ de los estudiantes la calificaron con 1 , el $3 \%$ de los estudiantes la evaluaron con 2, el 16\% de los estudiantes la apreciaron con 3, el 34\% de los estudiantes la estimaron con 4 y el $47 \%$ de los estudiantes la valoraron con 5 (ver Figura 13). El 97\% de los resultados muestran aceptación. 
Figura 13 - Resultados del grupo 704.

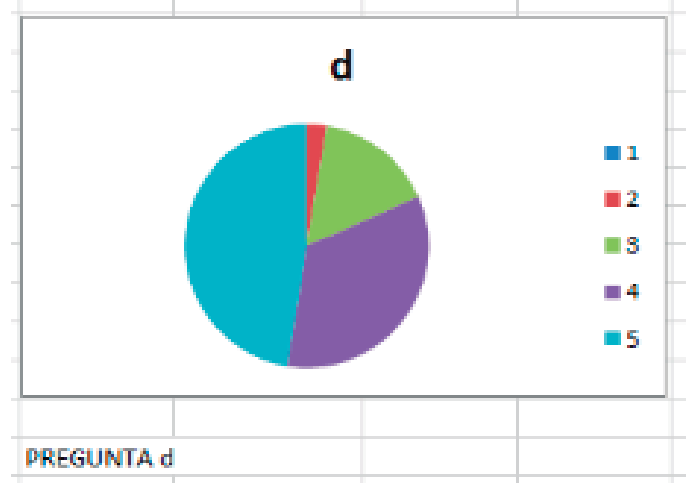

Fuente. Construcción de los autores.

¿Se sintió usted motivado a desarrollar los retos de forma natural y autónoma?, el 3\% de los estudiantes la califican con 1, el $5 \%$ de los estudiantes la calificaron con 2, el 26\% de los estudiantes la ponderaron con 3 , el $45 \%$ de los estudiantes la valoraron con 4 y el $21 \%$ de los estudiantes la consideraron con 5 (ver Figura 14). Los resultados muestran un 92\% de aceptación.

Figura 14 - Resultados del grupo 704.

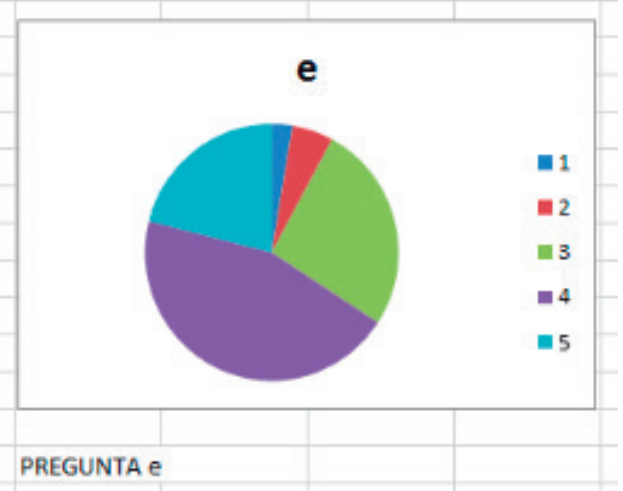

Fuente. Construcción de los autores.

\section{Consideraciones generales}

Los estudiantes demostraron gran expectativa por el desarrollo de las actividades en general. Fue retador para ellos subdividir una figura en regiones para evidenciar la equivalencia de fracciones mediante representaciones geométricas. Al explicar las inconsistencias de las pseudoparadojas se necesita que las fracciones asociadas a las piezas deban ser comparadas.

Para tal efecto, los criterios de comparación fueron establecidos en una línea recta inicialmente, pues los estudiantes aquí mostraron avances significativos. En un segundo momento emplearon la representación angular para las fracciones, pues esta representación fortalece argumentaciones para explicar las pseudoparadojas geométricas. 
Evidentemente durante el transcurso de las actividades aparecieron nuevos problemas geométricos que pusieron a prueba la habilidad matemática de los estudiantes y su comprensión matemática. El entorno de una clase tradicional fue modificándose gradualmente, con el propósito de hacer entender a los estudiantes, que estas actividades posibilitaron generar ambientes de trabajo más dinámicos a los que estaban habituados. Se dió importancia a la participación activa de los estudiantes y sus intervenciones surgieron de modo natural al lograr sustentar y explicar su proceder en cada actividad.

El docente fue un observador de las acciones de los estudiantes y contribuyó para guiar el trabajo original de los estudiantes. La intervención docente, en los momentos adecuados, permitió a los estudiantes ganar confianza para enfrentar, expresar y sustentar las soluciones. El eje central fue el estudiante mismo y mediante la generación de ambientes de trabajos dinámicos, mantenidos por la participación activa de los estudiantes, se apoyó la construcción de conocimiento.

Fue una oportunidad para concebir el aula en un espacio para la discusión, el respeto por las ideas y la construcción de conocimiento en comunidad. Se brindaron nuevas estrategias para fortalecer la comprensión de relaciones entre diferentes temáticas del grado séptimo, al aprovechar las inconsistencias de las pseudoparadojas. Los problemas enfrentados exigieron una mejor comprensión matemática al demandar de los estudiantes la búsqueda de nuevas conexiones para generar posibles soluciones.

Llama también la atención que el uso de diferentes materiales, para escudriñar propiedades de figuras geométricas, les permitió evidenciar relaciones que existen entre los triángulos, los rectángulos, los paralelogramos, los cuadrados, los rombos y los trapecios. Las transformaciones entre ellas les proporcionó de manera más sencilla el cálculo de áreas que usar tradicionalmente una fórmula. Esto reveló el concepto de área construido mediante apropiadas transformaciones en las figuras.

\section{Logros generales:}

- Las actividades fueron un reto para los estudiantes, debían resolverlas por ellos mismos, con la excepción de algunas cuestiones que debieron ser aclaradas por parte del docente. Las soluciones a cada una de las situaciones planteadas evidenciaron un desarrollo autónomo y responsable por cada grupo.

- El desarrollo de las actividades permitió que se origine la cooperación y la discusión entre los diferentes grupos de estudiantes. El eje motriz de las actividades, la socialización, permitió una mejor comunicación entre los estudiantes.

- Se desarrolló creatividad, al encontrar para una misma situación variadas soluciones y diferentes explicaciones. Los miembros participaron de diversas maneras en la solución de los problemas y se esforzaron por expresar las soluciones a los problemas planteados.

- Realizaron la transformación inversa de una figura en otra. De esta manera sustentaban que cuando una figura sufre transformaciones el área es igual. Se avanzó a tal grado, que la conservación del área es evidente a través de transformaciones. Manifestaron abiertamente que, si dos figuras son idénticas, ellas tienen la misma medida de área.

- El principio de la conservación del área les permitió realizar cálculos de área en figuras complejas de una manera sencilla. Dichos cálculos son un proceso de transformaciones en la figura. Aunque numéricamente se presentaron errores, fue evidente la capacidad de los estudiantes para transformar figuras. 
- Se avanzó con relación a la equivalencia entre fracciones. La representación angular, la hace más evidente, los puntos asociados están en la misma línea recta que pasa por el origen. Un progreso en el manejo del plano reticular es positivo, al observar como determinaron fracciones equivalentes y expresaron relaciones entre los numeradores y los denominadores.

- La razón, distancia vertical a distancia horizontal, apareció como determinante para establecer que una serie de puntos están sobre una misma línea recta.

- Se aportó otros elementos de mayor significatividad para los estudiantes, a través de la visualización, para fortalecer la construcción de sus conceptos matemáticos.

Dificultades generales. Se constatan las siguientes insuficiencias:

- Varios grupos de estudiantes no lograron subdividir una figura de manera adecuada para asociarle una fracción correspondiente a cada región. Por tanto, los problemas retadores, a pesar de no ser resueltos en su totalidad permitieron que exhibieran ánimo y destreza.

- La filmación de pequeños videos, para evidenciar las soluciones a determinadas preguntas de la actividad, generó un estado emocional en los estudiantes diferente a su comportamiento diario.

- En la representación angular para las fracciones, es la inclinación asociada al punto reticular la que permite establecer el orden en las fracciones positivas, contrario a lo que pensaron algunos estudiantes de la investigación (mientras más mida el segmento asociado más grande es la fracción).

- Limitaciones en establecer "la escritura correcta" para expresar que una fracción es mayor o menor que otra.

\section{CONCLUSIONES}

El proceso de investigación sobre las pseudoparadojas geométricas para potencializar el concepto de área en el grado séptimo, permitió dar respuesta al objetivo. Los resultados obtenidos resultaron esenciales en esta investigación, ellos son:

- Los diferentes planteamientos mostrados, en los cuales se precisan varias investigaciones sobre el estudio de la geometría y el uso de las pseudoparadojas para el aprendizaje de las matemáticas, en especial las que tiene ver con el área en las figuras geométricas, permitieron que las actividades planeadas demandaran de situaciones que desafiaran la comprensión matemática de los estudiantes.

- Al asumir en la investigación como marco teórico la resolución de problemas de Polya (1973), la visualización matemática de Zimmermann y Cunningham (1991), y la comunidad práctica de (WENGER, MCDERMOTT y SNYDER, 2002), para sustentar las actividades propuestas, se favoreció la construcción del significado de la conservación del área en los estudiantes del grado séptimo.

- El desarrollo de las actividades, en los diferentes grupos de trabajo, permitió el avance en los distintos tipos de pensamiento logrado por los estudiantes al realizar la evaluación de Ios análisis de los comentarios.

- Las actividades impactaron positivamente a los estudiantes durante todo el tiempo de su aplicación. Se percibió en su aplicación entusiasmo y disposición para aprender. El buen 
desempeño en las actividades se tuvo presente en los resultados de las encuestas realizadas, cuyos análisis confirmó el éxito de las actividades.

- Los resultados de las actividades, realizadas durante el primer y segundo periodo académico en la institución, constató que las matemáticas son agradables, dignas de estudiar y de profundizar en ellas, propiciando mejoras en el aprendizaje de los estudiantes para toda la vida. Estos resultados proyectaron y generaron saberes matemáticos que los estudiantes construyeron y negociaron por medio de la socialización.

- Los estudiantes lograron demostrar sus habilidades para enfrentar coherentemente los problemas que se plantean en las actividades, debido a que emplearon diferentes estrategias para dar cuenta del objetivo de cada actividad y se evidenciaron soluciones distintas para un mismo problema.

- Los estudiantes usaron adecuadamente las figuras en papel que recibieron y las transformaron en otras, estableciendo así que el área se conserva. De esta manera justifican la igualdad numérica del área.

- $\quad$ En los diferentes tipos de soluciones se evidencia creatividad por parte de los estudiantes para enfrentar cada actividad.

- $\quad$ El proceso de investigación basado en actividades conformadas por problemas retadores y que inviten y motiven al estudio de la matemática en los estudiantes de grado séptimo, de la Institución CEDID Ciudad Bolívar, permitió dar respuesta al objetivo.

\section{REFERENCIAS}

ARCAVI, A. The role of visual representations in the learning of mathematics. Educational Studies in Mathematics 52: 215-241. Kluwer Academic Publishers. Printed in the Netherlands, p. 217, 2003.

BERRÍ0, C. La conservación del área en las figuras geométricas a través del uso de paradojas en el séptimo grado. (Tesis de Maestría no publicada). Universidad Antonio Nariño, Bogotá, Colombia, 2015.

CASTRO, I.; PÉREZ, J. Las Paradojas en Matemáticas. Bogotá: Universidad Sergio Arboleda, 2002.

CONFERENCE OF EUROPEAN RESEARCH IN MATHEMATICS EDUCATION (CERME). Proceedings CERME 10. Irlanda. 2017. Disponible en: https://bit.ly/3gsOwRu. Acceso en: 23 abr. 2020.

CONFERENCE OF THE INTERNATIONAL GROUP FOR THE PSYCHOLOGY OF MATHEMATICS EDUCATION (PME). Proceedings of the First Regional Conference of the International Group for the Psychology of Mathematics Education. Rancagua, Chile. 2018. Disponible en: http://www.igpme.org/. Acceso en: 21 abr. 2020.

CONGRESO INTERNACIONAL DE EDUCACIÓN MATEMÁTICA (ICME). ICME-13 Monographs. Alemania. 2016. Disponible en: https://www.springer.com/series/15585. Acceso en: 25 abr. 2020.

DE GUZMÁN, M. El Rincón de la Pizarra. Cap. 0, el papel de la visualización. Pirámide, Madrid, p. 17, 2001.

DELORS, J. La Educación encierra un tesoro. Informe de la UNESCO de la Comisión internacional sobre la Educación para el siglo XXI, 1997. 
FALK, M. La enseñanza a través de problemas. Bogotá: Universidad Antonio Nariño. p. 16, 1980.

FARLOW, S. Paradoxes in Mathematics. DOVER PUBLICATIONS. Mineola, New York. United States, 2014.

FLORES, P. Paradojas Matemáticas Para La Formación De Profesores. Departamento de Didáctica de la Matemática, Campus Universitario de Cartuja, Universidad de Granada. SAEM THALES, s.f. Disponible en: https://bit.ly/2D91Yf9. Acceso en: 11 feb. 2019.

HERNÁNDEZ, R.; FERNÁNDEZ, C.; BAPTISTA, P. Metodología de la investigación (Vol. 3). Sexta edición. México: McGraw-Hill, 2014.

KILPATRICK, J. Problem solving in mathematics. Review of Educational Research, 39 (4), p. 523-534, 1969.

KLEINER, I.; MOVSHOVITZ, N. The Role of Paradoxes in the Evolution of Mathematics. Amer. Math. Monthy 688, December. 1994. Disponible en: https://bit.ly/2EDdCzi. Acceso en: 13 mar. 2019.

KONDRATIEVA, M. Understanding mathematics through resolution of paradoxes. Mediterranean Journal for Research in Mathematics Education, 6(1/2), p. 127-138. 2007.

LILJEDAHL, P.; SANTOS-TRIG0, M. Mathematical Problem Solving Current Themes, Trends, and Research. ICME-13 Topical Study Groups. Springer Nature Switzerland AG, 2019.

MACHO, M. Historias de matemáticas. Matemáticas a través de la paradoja. Revista de Investigación. Pensamiento Matemático. Vol II, número 2. 1 de octubre de 2012. Universidad del País Vasco, España, 2012. Disponible en: https:// bit.ly/3hTvFPV. Acceso en: 19 feb. 2019.

MINERVA, F. El proceso de investigación científica. Zulia, Venezuela: Universidad del Zulia, 2006.

MOVSHOVITZ-HADAR, N.; HADASS, R. More about mathematical paradoxes in preservice teacher education. Teaching \& Teacher Education. v. 7, n. 1. p. 79-92. Printed in Great Britain, 1991. Disponible en: https://bit.ly/2Pa2PhR. Acceso en: 23 feb. 2019.

MOVSHOVITZ-HADAR, N.; HADASS, R. Preservice Education of Math Teachers Using Paradoxes. Educational Studies in Mathematics 21: 265-287, 1990. Kluwer Academic Publishers. Printed in the Netherlands, 1990. Disponible en: https://bit.ly/33cPxJR. Acceso en: 15 abr. 2019.

PÉREZ, F. Olimpiadas Colombianas de Matemáticas para primaria 2000-2004. Bogotá: Universidad Antonio Nariño, 2004.

POCHULU, M.; RODRÍGUEZ, M. Educación Matemática. Aportes a la formación docente desde distintos enfoques teóricos. Buenos Aires. Argentina, 2012.

POLYA, G. How to solve it: A new aspect of mathematical method. Stanford University, Second Edition, Princeton University Press. Princeton, New Jersey, 1973.

POLYA, G. Mathematical Discovery: On understanding, learning, and teaching problem solving. Combined Edition. Stanford University. John Wiley \& Sons, 1981. 
ROJAS, 0. Modelo didáctico para favorecer la enseñanza - aprendizaje de la geometría con un enfoque desarrollador. Holguín. (Tesis doctoral no publicada). Universidad de Ciencias Pedagógicas José de la Luz y Caballero, 2009.

SCHOENFELD, A. H. Learning to think mathematically: Problem solving, metacognition, and sense making in mathematics. Handbook of research on mathematics teaching and learning, 334370, 1992.

SCHOENFELD, A. H. Mathematical problem solving. Elsevier. 2014.

SOUTO, B. Visualización en matemáticas un estudio exploratorio con estudiantes de primer curso de matemáticas. Facultad de Ciencias Matemáticas Universidad Complutense de Madrid. Departamento de Álgebra, 2009.

SRIRAMAN, B.; ENGLISH, L. Theories of Mathematics Education. New York: Springer, 2010.

SULLIVAN, M.; PANASUK, R. Fibonacci Numbers and an Area Puzzle: Connecting Geometry and Algebra in the Mathematics Classroom. School Science and Mathematics. Vol. 97 (3), March 1997. Disponible en: https://bit.ly/3gbNgCl. Acceso en: 15 abr. 2019.

WENGER, E. Comunidades de práctica. Aprendizaje, significado e identidad. Paidós. Barcelona, España, 2001.

WENGER, E.; MCDERMOTT, R.; SNYDER, W. Cultivating Communities of Practice. Harvard Business School Press. Boston, Massachusetts. Printed in the United States of America, 2002.

ZIMMERMANN, W.; CUNNINGHAM, S. (Eds.). Visualization in teaching and learning mathematics. Washington, USA: Mathematical Association of America, p. 67-76, 1991.

RECEBIDO EM: 24 jan. 2020

CONCLUÍDO EM: 4 mai. 2020 


\section{ANEXOS}

\section{Anexo 1: Encuesta de satisfacción a estudiantes}

Apreciado estudiante, ya terminamos las actividades, a partir de su experiencia como participante, responda las siguientes preguntas de 1 a 5, siendo cinco (5) la mayor calificación y uno (1) la menor calificación.

a) ¿Considera usted que las actividades desarrolladas motivan el estudio de la matemática?

$$
1 \square 2 \square 3 \square 4 \square 5 \square
$$

b) ¿Cree usted que su desempeño en el área de las matemáticas mejoraría si estas actividades se repitieran con frecuencia?

$$
1 \square 2 \square 3 \square 4 \square 5 \square
$$

c) ¿Las actividades propuestas constituyeron un reto para usted?

$$
1 \square 2 \square 3 \square 4 \square 5 \square
$$

d) ¿Considera usted que se vivió durante el desarrollo de las actividades un ambiente matemático?

$$
1 \square 2 \square 3 \square 4 \square 5 \square
$$

e) ¿Se sintió usted motivado a desarrollar los retos de forma natural y autónoma?

$$
1 \square 2 \square 3 \square 4 \square 5 \square
$$


